"This paper is a postprint of a paper submitted to and accepted for publication in [IET Generation, Transmission \& Distribution, 2019] and is subject to Institution of Engineering and Technology Copyright. The copy of record is available at the IET Digital Library". 


\title{
Simultaneous Reactive Power Sharing and Voltage Regulation in an Autonomous Networked Microgrid
}

\author{
Mohsen Eskandari ${ }^{1 *}, \operatorname{Li~Li~}^{1}$, Mohammad H. Moradi², Pierluigi Siano ${ }^{3}$, Frede Blaabjerg ${ }^{4}$ \\ ${ }^{1}$ Faculty of Engineering and Information Technology, University of Technology Sydney, Ultimo 2007, Sydney, \\ Australia \\ ${ }^{2}$ Department of Electrical Engineering, Bu-Ali Sina University, Hamedan, Iran \\ ${ }^{3}$ Department of Management \& Innovation Systems, University of Salerno, Salerno, Italy \\ ${ }^{4}$ Department of Energy Technology, Aalborg University, Aalborg, Denmark \\ *mohsen.eskandari@student.uts.edu.au
}

\begin{abstract}
Decentralized droop-like control method is the most favourable control system for power converter-based microgrids (MGs). In conventional $V$ - $Q$ droop loops, reactive power sharing is used as a means of voltage regulation to prevent currents from circulating among distributed generation units. However, since the voltage is not a global variable, reactive power sharing is not implemented precisely, and thus converters may be exposed to overcurrent conditions and the stability of the MGs is put at risk. Besides, the droop-like reactive power sharing causes voltage deviations and power quality issues. This paper proposes a novel control method which is able to implement accurate reactive power sharing and voltage regulation to its nominal band in a networked MG. Both the control targets are achieved, fast and simultaneously, by only one control signal. So the requirement of a secondary controller for voltage restoration is obviated. A novel power flowbased method is proposed to estimate the voltage at the MG main bus, which is adopted as a common variable, thus making the proposed method decentralized. The presented method is fast, effective and applicable to networked MGs with arbitrary topology. Simulation results prove the effectiveness and superiority of the proposed method over existing methods like the consensus control.
\end{abstract}

Index Terms - Distributed generation (DG), Microgrid (MG), Reactive power sharing, Renewable energy resources (RESs), Voltage regulation, Voltage source inverters (VSIs).

\section{Introduction}

Global warming and efficient energy source concerns have caused renewable energy sources (RESs) to become a growing trend in the field of energy [1]. The RESs are connected to the grid through power converter interfaces, and thus novel and advanced control schemes have been proposed in order to facilitate their integration into power systems [2][5]. Their intermittent nature, however, is a matter of concern, which has raised doubts about the reliability and security of RESs [6]. Experts and researchers, on the other hand, are attempting to address the issue. The most promising solution is the microgrid (MG) concept [7]. A number of distributed generation (DG) units including RESs, micro-sources and energy storage systems as well as loads, all located in a specific geographical (local) area, constitute a small grid. The MG concept is based on a control strategy and the corresponding infrastructure according to which this small grid is controlled in order to achieve the highest possible levels of the energy efficiency, power quality, security and reliability [8]-[9]. In addition, the MG idea enables this small grid to be operated in the islanded mode, isolated from the upstream network. This provides some great benefits for both utility companies and consumers [10]: 1) It improves the supply reliability to sensitive loads when the upstream grid is unavailable; 2) Energizing isolated rural and remote areas is feasible without installing new transmission lines (which causes extra costs and environmental concerns); 3) Black start would be more straightforward through an up-to-down (main grid) and down-to-up (MG) energizing, synchronizing and reconnecting process [11].

Islanded mode operation capability, however, is a challenging issue because of its state-of-the-art nature and lack of proper technology [12]. The most important aspect is developing a suitable control strategy for autonomous MGs. The hierarchical control structure consists of three levels [13]: 1) primary for power sharing and voltage regulation while securing the dynamic stability in transients, 2) secondary for the power quality improvement with larger time constant than the primary level, and 3) tertiary for energy management and optimization in the steady states.

The control system at the primary level of MGs is responsible for implementing active power sharing in order to realize energy management system (economic dispatch) or to dispatch power among DG units according to their available capacity [12]. Active power sharing is implemented accurately by employing a decentralized $f$ - $P$ droop-like control loop, as the frequency is a global variable throughout the MG [14].

Along with active power sharing, reactive power sharing should also be implemented among DG units in the MG for 
voltage regulation purpose [15]. The strategy in bulk power systems for voltage regulation is to keep the voltage magnitude at a constant value at generation buses via automatic voltage regulator (AVR) and the same strategy exists at load buses using flexible compensators. In MGs, however, because of the small value of the feeder impedance as well as feeders' impedance mismatch, a small error in the voltage magnitude poses the circulating current among converters, which in turn imposes extra power losses and also limits converters' and network's capacities [16]. To address this issue reactive power sharing is adopted as a means of voltage regulation to avoid circulating current. On the other hand, compensators may not support all the required reactive power, as the industrial loads working at a unit power factor can make the power system susceptible to resonance and harmonic problems. This makes DG units responsible for supporting the required reactive power. So, the accurate reactive power sharing must be established in the MG to prevent converters from overcurrent conditions.

The reactive power is generally controlled by the voltage magnitude via a $V-Q$ droop-based control loop. Nevertheless, it does not secure accurate reactive power sharing in MGs, as the voltage is a local variable. Besides, the $V$ - $Q$ droop control causes voltage deviations from the nominal value, and thus a secondary controller is required to restore the voltage profile to its nominal band. A lot of efforts have been done to address this issue in MGs [15]-[36]. These methods could be categorized as follows:

i. Signal injecting methods [17]-[18]; they affect the power quality and the response time is a matter of concern;

ii. Computational/optimized-based methods [19]-[20], which suffer from complexity and computational costs;

iii. Estimation-based methods [21]-[22], which are affected by the accuracy of the developed small-signal model, and also possess more sensitive stability margins;

iv. Virtual impedance-based methods [15]-[16], [20], [23][25]; although effective, they make the voltage regulation far worse and more complicated;

v. Parallel-based methods [16], [19], [26]-[31], which are not applicable to networked MGs. The stability analysis is not properly conducted since the interaction of droop controllers has not been taken into account;

vi. Distributed consensus methods [32]-[36], which need high band-width communication link.

To address the aforementioned issues related to reactive power sharing in MGs, the contributions of the proposed method in this paper are outlined as follows:

- A novel power flow method is proposed for droop-based isolated MGs to estimate the voltage magnitude and phase angle at generation buses as well as the power flowing through the feeders. Then, it is shown that the droop control causes a drastic voltage drop at the isolated MG's main bus, which has not been investigated in the literature;

- A supplementary control loop is proposed to add to the conventional $V$ - $Q$ droop control loop for accurate reactive power sharing and voltage regulation in autonomous networked MGs. The reactive power sharing is implemented immediately (as quickly as active power sharing) to prevent power converters from overcurrent conditions and protection system operations, which may lead to the instability and even collapse of MGs;
- Voltage regulation is executed at the same time with reactive power sharing so that the voltage deviation is restored simultaneously without requiring any extra control loop and additional efforts. Hence, the secondary controller is not needed for voltage restoration, while it is the drawback of the majority of other methods [15]-[36]. This issue is important in MGs due to random and frequent load fluctuations, to secure voltage stability and preserve voltage profile, thus retaining power quality;

- The corresponding gain of the supplementary loop is appropriately determined, considering the dynamic performance and stability, by developing a new statespace model in which the synergy of all the droop controllers in an MG is realized.

Moreover, the advantages of the suggested control system over the existing methods are as follows:

- The presented scheme is simple. It does not rely on complex computation or signal injecting methods [17][20]. Despite of the simplicity, which makes the plug-andplay capability feasible, the proposed method is effective;

- The proposed control scheme is applicable for parallel, series, parallel-series, and networked MGs, compared to many of the previous methods [17]-[31];

- The proposed method is fast to prevent power converters from being exposed to overcurrent conditions due to inaccurate reactive power sharing, which is a drawback of the methods in [17]-[22];

- The superiority of the method over the consensus control systems [32]-[36] is: 1) the response speed is faster; 2) only a lower communication bandwidth is required; 3 ) the secondary controller is not needed, while extra consensus protocol is required to restore the voltage in consensus control methods, which makes the required bandwidth higher; 4) it is more reliable and stable.

In Section 2, MG architecture and the conventional droop control system are described. The proposed method for reactive power sharing and voltage regulation is presented in Section 3. Dynamic stability by developing a novel smallsignal model is proposed and implemented in Section 4. Some simulation results are presented in Section 5 to prove that the proposed scheme is effective. Finally, conclusions are drawn in Section 6.

\section{Microgrid Design}

An MG consists of several parallel feeders that are connected to the point of common coupling (PCC). The PCC is the MG's main bus at which the MG is connected to the main grid via a circuit breaker. The feeders may include some DG units connected via voltage source inverters (VSI) as shown in Fig. 1. For the sake of reliability enhancement, DG units may be also connected to a meshed network. The MG central controller (MGCC) is responsible for the energy management in coordination with the upstream network operator. Optimal set-points or control (droop) gains are sent by the MGCC to dispatchable DG units via low band-width communication link (LBWCL). It should be clarified here that only the isolated operation of the MG is considered in developing the proposed control method. 


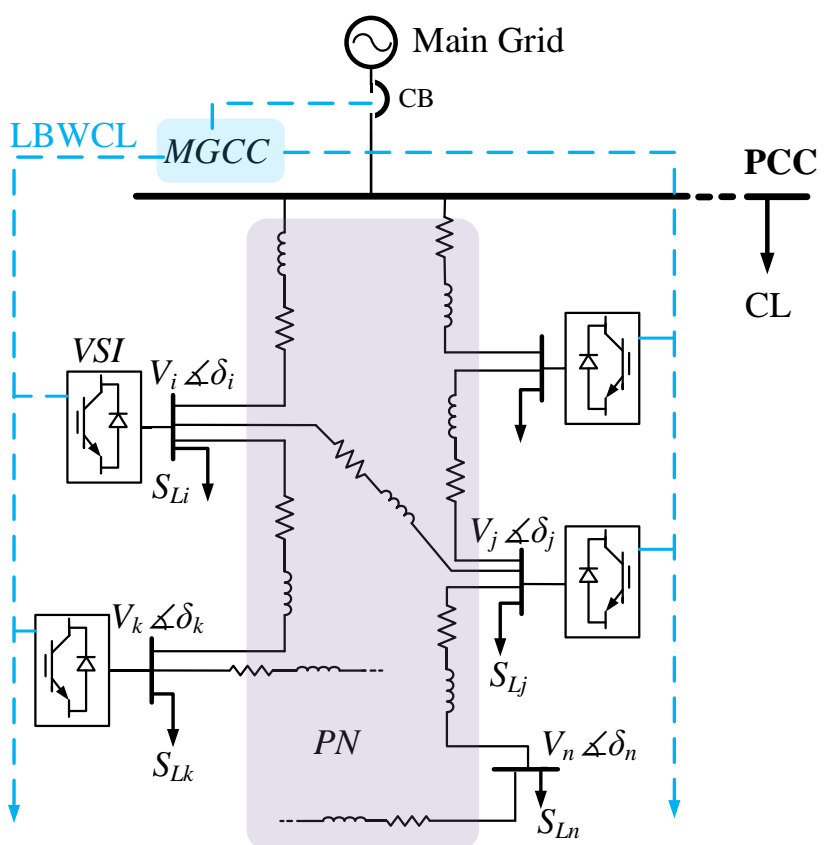

Fig. 1. MG topology, (PN: Power Network; CL: Common Load; CB: Circuit Breaker).

\subsection{Droop Control and Reactive Power Control Issue}

In a droop control, frequency $(f)$ and voltage magnitude $(V)$ are drooped according to the output active $(P)$ and reactive power $(Q)$, respectively. In order to investigate the relationship between $f \& P$ in the $f-P$ droop loop and $V \& Q$ in the $V$ - $Q$ droop loop, let us take $P_{i j}$ and $Q_{i j}$ flowing through the $i j^{\text {th }}$ electric power line at the $i^{\text {th }}$ node into consideration as:

$$
\begin{aligned}
& P_{i j}=\frac{V_{i}}{Z_{i j}}\left[\left(V_{i}-V_{j} \cos \left(\delta_{i j}\right)\right) \quad V_{j} \sin \left(\delta_{i j}\right)\right]\left[\begin{array}{ll}
\cos \left(\theta_{i j}\right) & \sin \left(\theta_{i j}\right)
\end{array}\right]^{T}
\end{aligned}
$$

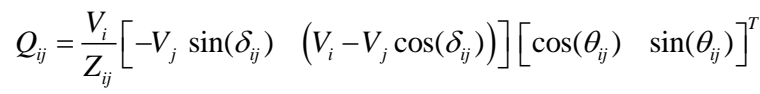

where the $i$ and $j$ indices denote the $i^{t h}$ and $j^{\text {th }}$ nodes; $V$ and $\delta$ are the magnitude and phase angle of the voltage at the end nodes, respectively; $Z$ and $\theta$ are the amplitude and phase angle of the feeder impedance. Although MGs are located in the low-voltage grids in which the $\mathrm{X} / \mathrm{R}$ ratio of the feeder impedance is not too high, the overall $\mathrm{X} / \mathrm{R}$ ratio of the feeder is high enough because of installing LCL filters, isolating transformers and virtual inductance. In the power network with dominant inductive line impedance $\left(\theta \approx 90^{\circ}\right)$, the power flow equations in (1)-(2) are updated as:

$$
\begin{aligned}
& P_{i j}=\frac{V_{i} V_{j} \sin \left(\delta_{i j}\right)}{X_{i j}} \\
& Q_{i j}=\frac{V_{i}}{X_{i j}}\left(V_{i}-V_{j} \cos \left(\delta_{i j}\right)\right)
\end{aligned}
$$

where $X_{i j}$ is the $i j^{t h}$ interconnecting line reactance. Due to the sensitivity of $P$ to the phase angle difference $\left(\delta_{i j}\right)$ and $Q$ to the voltage magnitude difference $\left(V_{i}-V_{j}\right)$ at end nodes, the droop equations at node $i$ are specified as [24]:

$$
\begin{aligned}
& \omega_{i}=\omega_{0}-k_{p i} P_{i} \\
& \left\{\begin{array}{l}
v_{\text {odrefi }}=V_{0}-k_{q i} Q_{i} \\
v_{\text {oqrefi }}=0
\end{array}\right.
\end{aligned}
$$

where $\omega\left(\omega_{0}\right)$ is the operating (nominal) angular frequency; $v_{\text {odref }} \& v_{\text {oqref }}$ are the reference values for direct and quadrature $(d-q)$ components of the VSI voltage, respectively; $V_{0}$ is the nominal voltage magnitude; $k_{p} \& k_{q}$ are the droop gains and $P$ and $Q$ are the average of output active and reactive power which are given after passing instantaneous active and reactive power $(p \& q)$ through a low-pass filter (LPF), and the index $i$ denotes the $i^{\text {th }}$ generation bus. Power sharing is implemented by adjusting the droop gains related to $f-P$ droop controllers according to the DG unit size to share the demanded power among DG units according to their capacity. The $f-P$ loop droop gains may also be determined by the MGCC for the optimal dispatch. In either cases, the following equilibrium is achieved:

$$
k_{p 1} P_{1}=k_{p i} P_{i} \text { for } i=2: n
$$

Since the frequency is a global variable (in this work the expression of global variable means that its magnitude is same throughout the MG in steady states), the active power sharing is implemented precisely [14].

The strategy for the reactive power sharing is to make the converters' contributions in supporting the reactive power according to their capacity to prevent them from overcurrent condition. It is executed by assigning the $V$ - $Q$ droop gains at each generation bus so that the equilibrium point given in (8) is established [16].

$$
k_{q 1} Q_{1}=k_{q i} Q_{i} \text { for } i=1: n
$$

Furthermore, the reactive power sharing implementation prevents the reactive current from circulating among VSIs. Nevertheless, the reactive power sharing is not implemented as accurately as the active power sharing [24]-[28]. The problem is that the voltage is a local variable because of the voltage drop through the feeder impedance (voltage drop makes the voltage magnitude vary from point to point over the feeder because of the flowing power) [31]. Besides, the sensitivity of the reactive power to the voltage magnitude is not as high as the sensitivity of the active power to the phase angle.

\subsection{Droop Control and Voltage Regulation Issue}

In addition to reactive power sharing issue, voltage deviation from the nominal band is another problem related to the $V-Q$ droop control rule given in (6). This in turn raises the power quality issue which requires adopting a secondary controller for voltage restoration [12], [13], [32]. The other issue is the dramatic voltage drop at the MG's main bus (PCC, see Fig. 1) at which the common loads of MG are connected. In order to investigate this issue, suppose that $n$ DG units are connected to the PCC via a feeder, as shown in Fig. 2(a). From the $i^{\text {th }}$ DG point of view, the voltage drop over the $i j^{\text {th }}$ feeder in the $d-q$ reference frame is obtained as [31]: 


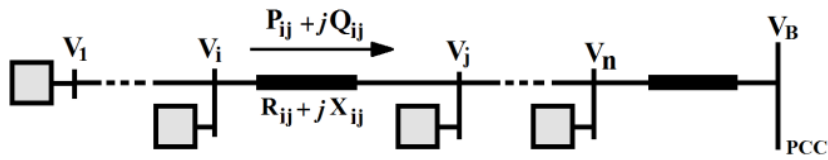

$a$

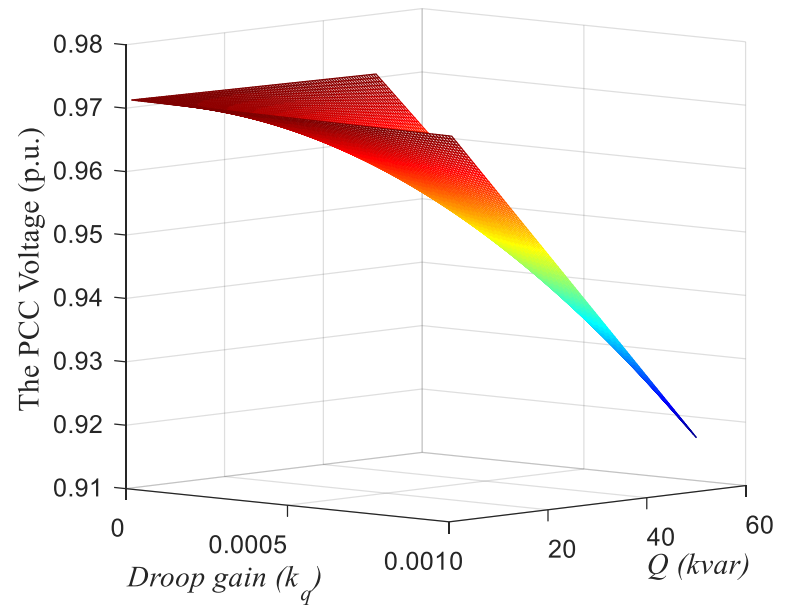

$b$

Fig. 2. (a) A feeder connecting DG units to PCC, (b) voltage drop over the feeder consisting of three DG units.

$\Delta v_{d i j}=v_{o d i}-v_{o d j}^{(i)}=R_{i j} i_{d i j}-X_{i j} i_{q i j}$

$\Delta v_{q i j}=v_{o q i}-v_{o q j}^{(i)}=R_{i j} i_{q i j}+X_{i j} i_{d i j}$

where $R_{i j} \& X_{i j}$ denote the resistance and reactance of the $i j^{\text {th }}$ feeder, $\Delta V_{d i j} \& \Delta V_{q i j}$ are the voltage drop over the $d$ - $q$ axis, $i_{d i j}$ $\& i_{q i j}$ are the $d-q$ component of the current, $v^{(i)}{ }_{o d j} \& v^{(i)}{ }_{o q j}$ are the $d$ - $q$ components of the voltage at bus $j$ in the reference frame of bus $i . P_{i j} \& Q_{i j}$ passing through the feeder are given as:

$P_{i j}=v_{o d j} i_{d i j} \quad \& \quad Q_{i j}=-v_{o d j} i_{q i j}$

It is supposed that $v_{o q i}$ is zero, according to (6). Substituting $i_{d i j} \& i_{q i j}$ from (11) into (9)-(10) the quadratic voltage equation over the feeder is developed:

$v_{o d j}^{(i) 2}-v_{o d j}^{(i)} v_{o d i}+R_{i j} P_{i j}+X_{i j} Q_{i j}=0$

$v_{o d j}^{(i)} v_{o q j}^{(i)}-R_{i j} Q_{i j}+X_{i j} P_{i j}=0$

$v^{(i)}{ }_{\text {odj }}$ is obtained by solving the quadratic equation (12) as:

$v_{o d j}^{(i)}=\frac{v_{o d i}+\sqrt{v_{o d i}^{2}-4\left(R_{i j} P_{i j}+X_{i j} Q_{i j}\right)}}{2}$

$v^{(i)}{ }_{o q j}$ is obtained by substituting $v^{(i)}{ }_{o d j}$ into (13). The phase angle at bus $j$ is obtained as:

$$
\delta_{j}^{(i)}=\arctan \left(v_{o q j}^{(i)} / v_{o d j}^{(i)}\right)=\delta_{j}-\delta_{i}=-\delta_{i j}
$$

The following transformation matrix is used to obtain the $d-q$ component of voltage at bus $j$ in the $d-q$ reference frame of bus $j$ : $\left[\begin{array}{l}v^{(j)}{ }_{o d j} \\ v^{(j)}{ }^{o q j}\end{array}\right]=\Gamma\left(\delta_{i j}\right)\left[\begin{array}{l}v_{o d j}^{(i)} \\ v_{o q j}^{(i)}\end{array}\right]$

where $\Gamma\left(\delta_{i j}\right)=\left[\begin{array}{cc}\cos \left(\delta_{i j}\right) & -\sin \left(\delta_{i j}\right) \\ \sin \left(\delta_{i j}\right) & \cos \left(\delta_{i j}\right)\end{array}\right], \delta_{i j}=\delta_{i}-\delta_{j}$

Consequently, the voltage at the MG main bus (PCC) is given as:

$$
v^{(n)}{ }_{o d B}=\frac{v^{(n)}{ }_{\text {odn }}+\sqrt{v^{(n)}{ }^{2}{ }^{2}-4\left(R_{n B} P_{n B}+X_{n B} Q_{n B}\right)}}{2}
$$

where

$v_{o d n}^{(n-1)}=\frac{v_{o d(n-1)}^{(n-1)}+\sqrt{v_{o d(n-1)}^{(n-1)}-4\left(R_{(n-1) n} P_{(n-1) n}+X_{(n-1) n} Q_{(n-1) n}\right)}}{2}$

$v^{(n-2)}{ }_{o d(n-1)}=\frac{v^{(n-2)}{ }_{o d(n-2)}+\sqrt{v^{(n-2)}{ }_{o d(n-2)}{ }^{2}-4\left(R_{(n-2)(n-1)} P_{(n-2)(n-1)}+X_{(n-2)(n-1)} Q_{(n-2)(n-1)}\right)}}{2}$

$\vdots$

$V_{i}=V_{0}-k_{q i} Q_{i}$

The unknown variables are $P_{(n-2)(n-1)}$ and $P_{(n-2)(n-1)}(\forall n>i+2)$ which could be obtained according to the droop control rules as:

$$
\begin{aligned}
P_{(n-2)(n-1)}= & P_{i(i+1)}+\left(\sum_{j=i+1}^{n-2} \frac{k_{p i}}{k_{p j}}\right) P_{i} \\
& -\left(\sum_{j=i}^{n-3} R_{(j)(j+1)}\left(\frac{P_{(j)(j+1)}^{2}+Q_{(j)(j+1)}^{2}}{v_{o d j}^{2}}\right)\right), \forall n>i+2 \\
Q_{(n-2)(n-1)}= & Q_{i(i+1)}+\sum_{j=i+1}^{n-2}\left(\frac{V_{0}-v_{o d j}^{(j)}}{k_{q j}}\right) \\
& -\left(\sum_{j=i}^{n-3} X_{(j)(j+1)}\left(\frac{P_{(j)(j+1)}^{2}+Q_{(j)(j+1)}^{2}}{v_{o d j}^{2}}\right), \forall n>i+2\right.
\end{aligned}
$$

Equation (18) is developed based on the fact that active power sharing is implemented accurately according to the droop gains in (7). Eq. (19) is developed from the $V$ - $Q$ droop rule in (6) provided that the VSI inner control loops follow the reference value very fast (with faster dynamic than power control), which is the case [12]. The second sigma (the third term at the right-hand side) in (18) and (19) denotes the power losses through the feeders' impedance. By substituting (18)(19) in (17), $V_{B}$ is obtained from the local measurements that are $P_{i j} \& Q_{i j}$.

Fig. 2(b) shows the voltage variation at the MG main bus $\left(V_{B}\right)$ which is connected to a feeder composed of three DG units. The voltage variation is drawn versus the reactive power and droop gain variations. Large droop gains (which may help improve the accuracy of $Q$-sharing) in severe load conditions lead to drastic voltage drops at the MG main bus which must be considered in the control scheme.

\section{The Proposed Method}

The corresponding $V$ - $Q$ droop control equations for $i^{t h} \& j^{\text {th }}$ DG units are given as follows:

$V_{i}=V_{0}-k_{q i} Q_{i}$ 
$V_{j}=V_{0}-k_{q j} Q_{j}$

$V_{i}$ and $V_{j}$ are the output voltage magnitudes of DG $i$ and DG $j$, respectively, at equilibrium points which are determined by droop controllers. Subtracting both sides of (21) from (20) results in:

$$
V_{i}-V_{j}=k_{q j} Q_{j}-k_{q i} Q_{i}
$$

In the operating condition with a conventional $V$ - $Q$ droop control loop, $V_{i}$ is not equal to $V_{j}$ because of the voltage drop, and thus the right-hand side of (22) is not equal to zero, that is, $k_{q i} Q_{i} \neq k_{q j} Q_{j}$. Therefore, accurate reactive power sharing is not achieved, according to (8). So, the conventional $V$ - $Q$ droop rule must be modified for reactive power sharing purposes. In the next subsection, a simple and effective method is developed for reactive power sharing and voltage regulation in MGs by adding a supplementary control loop to the conventional $V$ - $Q$ droop control loop.

Reactive power sharing: to obtain accurate reactive power sharing a common variable (a reference variable) is needed to coordinate droop controllers. The MG's main bus voltage is assumed as a common variable to coordinate $V-Q$ control loops in an MG. $V-Q$ control loops of DG $i$ and DG $j$ are modified by adding a supplementary control signal $(\alpha)$ to the droop loop as the following:

$$
\begin{aligned}
& V_{i}=V_{0}-k_{q i} Q_{i}+\alpha_{i} \\
& V_{j}=V_{0}-k_{q j} Q_{j}+\alpha_{j}
\end{aligned}
$$

Subtracting both sides of (24) from (23) results:

$$
V_{i}-V_{j}=k_{q j} Q_{j}-k_{q i} Q_{i}+\alpha_{i}-\alpha_{j}
$$

It is assumed that accurate reactive power sharing is implemented by adding the additional control signals so that $k_{q i} Q_{i}=k_{q j} Q_{j}$. Then (25) is updated as:

$V_{i}-V_{j}=\alpha_{i}-\alpha_{j}$

The next step is to determine the supplementary control signals so that (26) is reachable. The following supplementary control signal is proposed:

$$
\alpha_{i}=\left\{\begin{array}{ccc}
k_{s}\left[\int\left(V_{i}-V_{B}\right) d t\right] & \text { if } & k_{s}\left[\int\left(V_{i}-V_{B}\right) d t\right]<V_{i}-V_{B} \\
V_{i}-V_{B} & \text { if } & k_{s}\left[\int\left(V_{i}-V_{B}\right) d t\right] \geq V_{i}-V_{B}
\end{array}\right.
$$

where $V_{B}$ is the voltage magnitude of the MG main bus, and $k_{s}$ is the supplementary loop gain. It can be seen that, at the steady state, we have

$$
\alpha_{i}=V_{i}-V_{B}
$$

By replacing (28) into (26) the equality in (26) is established as the following:

$$
V_{i}-V_{j}=\left(V_{i}-V_{B}\right)-\left(V_{j}-V_{B}\right)
$$

Note that the integrator term in (27) is included to make the time constant of the supplementary loop larger than droop controller; otherwise, the time constant of the supplementary loop would be less than the droop control loop, which makes the system unstable. To get the time constant of the droop control loop, let take the LPF dynamic into account in the $V$ $Q$ droop control rule as following:

$V_{i}=V_{0}-k_{q i} \frac{1}{\tau s+1} q_{i}+\alpha_{i}$

where $\tau=1 / \omega_{c}$ is the time constant of the LPF and $\omega_{c}$ is its cutting frequency. The time constant of the supplementary loop must be larger than $\tau$, otherwise it is in conflict with the droop loop.

Eq. (27) is implemented by adopting a sample and hold $(\mathrm{S} \& \mathrm{H})$ block, as shown in Fig. 3. As long as the I/O signal of the $\mathrm{S} \& \mathrm{H}$ block is 1 , it follows the input value. When the $\mathrm{I} / \mathrm{O}$ signal turns to 0 , the $S \& H$ block holds the last input value. In this way, the output value of the $\mathrm{S} \& \mathrm{H}$ block is saved as the control signal $(\alpha)$ in the steady state and the integrator is reset when the next transient is reached. The voltage magnitudes, $V_{i}$ and $V_{B}$ are realized by obtaining the direct component of measured voltage at $\mathrm{VSI}_{i}$ 's output and PCC bus, respectively. As $V_{B}$ is a DC value, the existing LBWCL is sufficient to distribute it to DG units. This technique is applicable to all DG units in the MGs and its performance is independent from the configuration of the MG. Nevertheless, the novel method presented in Section 2.2 for $V_{B}$ estimation could be used to improve the reliability of the control system whenever the LBWCL is not available. It also makes the proposed method a decentralized one as it is only based on local measurements.

Voltage regulation: applying the proposed method, at the steady state, to the droop control rule at the $n^{\text {th }} \mathrm{DG}$ unit in Fig. 2(a), results in:

$V_{n}=V_{0}-k_{q n} Q_{n}+V_{n}-V_{B}$

From (30) $V_{B}$ is given as:

$V_{B}=V_{0}-k_{q n} Q_{n}$

which is independent from the voltage at the MG generation buses and could be adjusted by tuning the droop gains. On the other hand, from (17) we have:

$v_{o d B}^{(n)}=\frac{v_{o d n}^{(n)}+\sqrt{v_{o d n}^{(n)}{ }^{2}-4\left(R_{n B} P_{n B}+X_{n B} Q_{n B}\right)}}{2}$

Due to the small voltage angle difference between bus $n\left(V_{n}\right)$ and PCC $\left(V_{B}\right)$, we have:

$$
v_{o d B}^{(n)} \approx V_{B}
$$

From (31-33), the voltage at bus $n$ would be:

$$
V_{n}=2 V_{0}-2 k_{q n} Q_{n}-\sqrt{V_{n}^{2}-4\left(R P_{n}+X Q_{n}\right)}
$$

Taking the partial derivative of the both sides of (34) versus $Q_{n}$ we obtain:

$$
\frac{\partial V_{n}}{\partial Q_{n}}=-2 k_{q n}+\frac{2 X}{\sqrt{V_{n}^{2}-4\left(R P_{n}+X Q_{n}\right)}}
$$

Noting that $V_{n}^{2} \gg 4\left(R P_{n}+X Q_{n}\right)$ and $V_{n} \approx V_{0}$ updated as: 


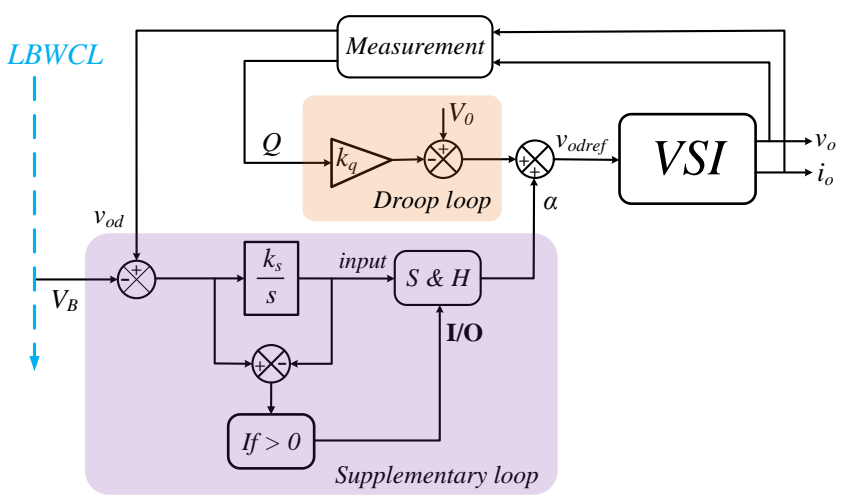

a

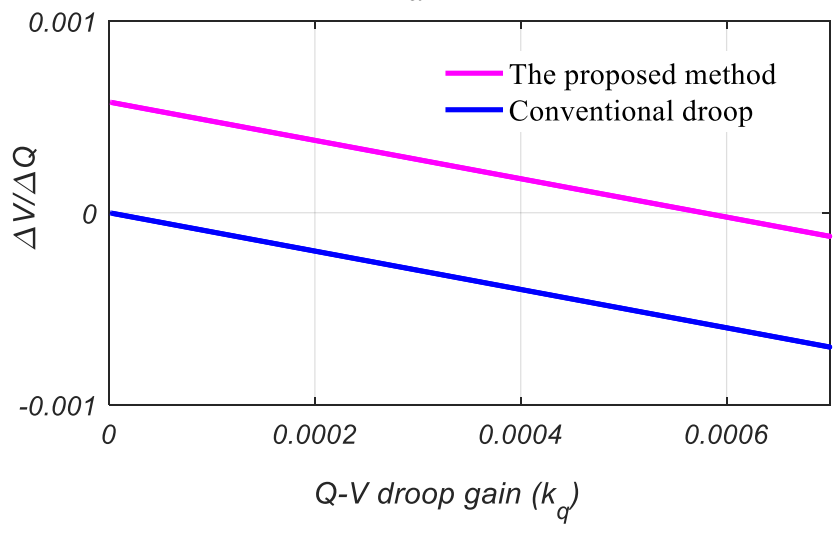

(b)

Fig. 3. The proposed method, (a) the supplementary control loop for reactive power sharing, (b) $\Delta V / \Delta Q$ which is positive for a variety of droop gains and can be regarded as virtual boost $V$-Q loop

$$
\frac{\partial V_{n}}{\partial Q_{n}} \approx-k_{q n}+\frac{X}{V_{0}}
$$

Eq. (36) specifies voltage variation with respect to the droop gain depicted in Fig. 3(b), which is positive in a wide range of droop gains. It shows that the proposed method converts the $Q-V$ droop control to a virtual $Q-V$ boost control that restores the voltage magnitude to the nominal band, while the accurate reactive power sharing is achieved.

\section{Dynamic Stability}

The next step is to determine the integrator gains of the supplementary control loops so that desirable dynamic performances are obtained. The performance of the droop control relies on communicating control signals (voltage and frequency) through the power network based on the power network variables, i.e. $P$ and $Q$. Consequently, droop controllers interrelate to each other through the power network. Hence, tuning control parameters based on the model of only one DG unit would not be accurate and all the droop controllers as well as the power network and their interactions should be inserted into the model. To this end, a state-space model of the MG is adopted to appropriately design the control parameters as presented in the next subsection. To develop a complete state-space model of the MG firstly a small-signal model of individual DG units is obtained. Then the power network is modeled so that DG units' models are correlated.
1) Individual DG unit Model: state-space model of the $i^{\text {th }} \mathrm{DG}$ unit is obtained by developing small-signal model of the following parts:

Droop controller: from (5) the phase angle dynamic is obtained as:

$$
\dot{\delta}_{i}=-k_{p i} P_{i}
$$

An LPF $\omega_{c} /\left(s+\omega_{c}\right)$ is used to obtain the average active and reactive power $P$ and $Q$. As a result two more states are added to the system:

$$
\begin{aligned}
& \dot{P}_{i}=-\omega_{c} P_{i}+\omega_{c} p_{i} \quad \text { where } \quad p_{i}=\left[\sum_{j=1}^{n}\left(\sigma_{i j} p_{i j}\right)+p_{\text {LDConi }}\right] \\
& \dot{Q}_{i}=-\omega_{c} Q_{i}+\omega_{c} q_{i} \quad \text { where } \quad q_{i}=\left[\sum_{j=1}^{n}\left(\sigma_{i j} q_{i j}\right)+q_{L D C o n i}\right]
\end{aligned}
$$

where the $i$ and $j$ indices denote the $i^{\text {th }} \& j^{\text {th }}$ buses, respectively, $n$ is the number of MG buses, $p_{i j} \& q_{i j}$ are the active and reactive powers, $p_{L D C o n i} \& q_{L D C o n i}$ are the constant active and reactive loads, and $\omega_{c}$ is the cutting frequency of the LPF. $\sigma_{i j}$ is 1 if bus $i$ is connected to bus $j$ and 0 otherwise.

VSI Model: VSI consists of two nested voltage and current control loops [12]. State variables of the voltage control loop (outer loop) are defined as:

$$
\dot{\phi}_{d i}=v_{\text {odrefi }}-v_{\text {odi }} \quad \& \quad \dot{\phi}_{q i}=v_{\text {oqrefi }}-v_{o q i}
$$

where $v_{o d} \& v_{o q}$ are the $d-q$ components of the measured output voltage, $v_{\text {odref }} \& v_{\text {oqref }}$ are defined by the droop control and virtual impedance loops as the following:

$$
\begin{aligned}
& v_{\text {odrefi }}=V_{0}-k_{q i} Q_{i}-R_{v i} i_{\text {odi }}+\omega_{0} L_{v i} i_{\text {oqi }} \\
& v_{\text {oqrefi }}=-R_{v i} i_{\text {oqi }}-\omega_{0} L_{v i} i_{\text {odi }}
\end{aligned}
$$

where $R_{v} \& L_{v}$ are the virtual resistance and inductance, respectively, and $i_{o d} \& i_{o q}$ are the $d-q$ components of DG unit output current given as in (43):

$\left[\begin{array}{l}i_{o d i} \\ i_{o q i}\end{array}\right]=\left(\sum_{j=1}^{n} \sigma_{i j}\left[\begin{array}{l}i_{d i j} \\ i_{q i j}\end{array}\right]\right)+\left[\begin{array}{l}i_{L D C o n d i} \\ i_{L D C o n q i}\end{array}\right]$

where $i_{d i j} \& i_{q i j}$ are the $d-q$ components of the current from bus $i$ to bus $j$ and $i_{L D C o n d i} \& i_{L D C o n q i}$ are $d-q$ components of local load current considered as disturbance. The equations representing proportional-integrator (PI) control loops are [37]:

$$
\begin{aligned}
& i_{\text {fdrefi }}=F i_{\text {odi }}-\omega_{0} C_{f i} v_{o q i}+k_{P v i} \dot{\phi}_{d i}+k_{I v i} \phi_{d i} \\
& i_{\text {fqrefi }}=F i_{o q i}+\omega_{0} C_{f i} v_{o d i}+k_{P v i} \dot{\phi}_{q i}+k_{I v i} \phi_{q i}
\end{aligned}
$$

where $k_{P v} \& k_{I v}$ are the proportional and integral gains of PI voltage controller, respectively, $C_{f}$ is the LC filter capacitance, and $F$ is the feedforward gain. For the current control loop, the 0 state variables are defined as:

$$
\dot{\gamma_{d i}}=i_{f d r e f i}-i_{f d i} \quad \& \quad \dot{\gamma}_{q i}=i_{f q r e f i}-i_{f q i}
$$

where $i_{\text {fdref }} \& i_{\text {fqref }}$ are defined by the voltage control loop in (44)-(45) and $i_{f d} \& i_{f q}$ are the $d-q$ components of LC filter inductor currents, respectively.

LC Filter Model: the differential equations representing 
dynamic of the LC filter are acquired in $d-q$ form as [37]:

$\dot{i_{f d i}}=-\frac{r_{f i}}{L_{f i}} i_{f d i}+\omega_{i} i_{f q i}+\frac{1}{L_{f i}} v_{I d i}-\frac{1}{L_{f i}} v_{o d i}$

$\dot{i_{f q i}}=-\frac{r_{f i}}{L_{f i}} i_{f q i}-\omega_{i} i_{f d i}+\frac{1}{L_{f i}} v_{I q i}-\frac{1}{L_{f i}} v_{o q i}$

$\dot{v_{o d i}}=\omega_{i} v_{o q i}+\frac{1}{C_{f i}} i_{f d i}-\frac{1}{C_{f i}} i_{o d i}$

$\dot{v_{o q i}}=-\omega_{i} v_{o d i}+\frac{1}{C_{f i}} i_{f q i}-\frac{1}{C_{f i}} i_{o q i}$

where $\omega$ is the operating angular frequency determined by the droop control (1), $R_{f}, L_{f}$, and $C_{f}$ are the resistance, inductance and capacitance of LC filter respectively, and $v_{I d} \& v_{I q}$ are the $d-q$ components of reference voltage which are the input to the converter [37]:

$v_{I d i}=-\omega_{0} L_{f i} i_{f q i}+k_{P c i} \dot{\gamma}_{d i}+k_{I c i} \gamma_{d i}+v_{o d i}$

$v_{I q i}=\omega_{0} L_{f i} i_{f d i}+k_{P c i} \dot{\gamma}_{q i}+k_{I c i} \gamma_{q i}+v_{o q i}$

where $k_{P_{c}} \& k_{I c}$ are the proportional and integrator gains of PI current controller, respectively, $L_{f}$ is the LC filter inductance.

2) Power Network Model: operation of the droop controllers at MG nodes are correlated to each other through the power network via power flow. So in order to model the power network properly, $p$ and $q$ should be developed as functions of voltage magnitude and phase angle. In this way, DG unit models are correlated and their interaction is realized properly. To this end, the current from bus $i$ to bus $j$ through $i j^{\text {th }}$ power line $\left(Z_{i j} \measuredangle \theta_{i j}\right)$ is obtained as:

$\left[\begin{array}{l}i_{d i j} \\ i_{q i j}\end{array}\right]=\frac{1}{Z_{i j}} \Gamma\left(-\theta_{i j}\right)\left[\begin{array}{l}v_{o d i}-v_{o d j}^{(i)} \\ v_{o q i}-v_{o q j}^{(i)}\end{array}\right]$

where $\Gamma$ operator is given from (16), and $v_{o d q i} \& v^{(i)}$ odqj are $d$ $q$ components of voltage at bus $i$ and bus $j$, respectively. However, according to the frequency droop rule (37) there are different reference frames at $i \& j$ buses in transients. So (53) is updated as:

$$
\left[\begin{array}{c}
i_{d i j} \\
i_{q i j}
\end{array}\right]=\frac{1}{Z_{i j}}\left(\Gamma\left(-\theta_{i j}\right)\left[\begin{array}{l}
v_{o d i} \\
v_{o q i}
\end{array}\right]-\Gamma\left(\delta_{j i}-\theta_{i j}\right)\left[\begin{array}{l}
v_{o d j} \\
v_{o q j}
\end{array}\right]\right)
$$

where $\Gamma\left(\delta_{i j}\right)$ is reference frame transformation matrix, and $v_{o, d q j}$ is the $d-q$ components of voltage at bus $j$ in the local reference frame of bus $j$. Accordingly, the power flowing from bus $i$ to bus $j$ is obtained as in (56) by substituting (54) into (55):

$$
\begin{aligned}
& {\left[\begin{array}{l}
p_{i j} \\
q_{i j}
\end{array}\right]=\left[\begin{array}{cc}
v_{o d i} & v_{o q i} \\
v_{o q i} & -v_{o d i}
\end{array}\right]\left[\begin{array}{l}
i_{d i j} \\
i_{q i j}
\end{array}\right]} \\
& {\left[\begin{array}{l}
p_{i j} \\
q_{i j}
\end{array}\right]=\frac{1}{Z_{i j}}\left[\begin{array}{cc}
v_{o d i} & v_{o q i} \\
v_{o q i} & -v_{o d i}
\end{array}\right]\left(\Gamma\left(-\theta_{i j}\right)\left[\begin{array}{c}
v_{o d i} \\
v_{o q i}
\end{array}\right]-\Gamma\left(\delta_{j i}-\theta_{i j}\right)\left[\begin{array}{l}
v_{o d j} \\
v_{o q j}
\end{array}\right]\right)}
\end{aligned}
$$

Consequently, $i_{d i j} \& i_{q i j}$ in (43) and $p_{i j} \& q_{i j}$ in (38)-(39) are updated by (54) and (56), respectively. Active and reactive power related to constant loads, $p_{\text {LDConi }} \& q_{\text {LDConi }}$ in (38)-(39), are developed based on (55) as function of bus voltage and constant currents, $i_{L D C o n d i} \& i_{L D C o n q i}$, as disturbance.
3) MG model: the state-space model of the whole MG is obtained by linking the state-space model of individual DG units via the power network model given in (54) and (56) as the following:

$$
\dot{x_{M G}}=A_{M G} x_{M G}+B_{M G} u_{M G}
$$

$A_{M G}=\operatorname{diag}\left[A_{D G 1} \ldots A_{D G i} \ldots A_{D G n}\right]+\sum \sigma_{i j} A_{D G i j} \quad \forall i, \quad j=1: n, \quad j \neq i$

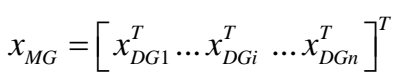

$x_{D G i}=\left[\Delta \delta_{i} \Delta \tilde{P}_{i} \Delta \tilde{Q}_{i} \Delta \phi_{d i} \Delta \phi_{q i} \Delta \gamma_{d i} \Delta \gamma_{q i} \Delta i_{f_{d i}} \Delta i_{f_{q i}} \Delta v_{o_{d i}} \Delta v_{o_{q i i}}\right]^{T}$

$u_{M G}=\left[u_{D G 1}^{T} \ldots u_{D G i}^{T} \ldots u_{D G n}^{T}\right]^{T}$

$u_{D G i}=\left[\begin{array}{ll}\Delta i_{\text {LDCondi }} & \Delta i_{L D C o n q i}\end{array}\right]^{T}$

$B_{M G}=\operatorname{diag}\left[B_{D G 1} \ldots B_{D G i} \ldots B_{D G n}\right]$

$B_{D G i}=\left[\begin{array}{lll}B_{D r p i}^{T} & B_{V S I i}^{T} & B_{L C f i}^{T}\end{array}\right]^{T}$

$B_{D r p i}=\left[\begin{array}{ccc}0 & \omega_{c} & 0 \\ 0 & 0 & \omega_{c}\end{array}\right]^{T}\left[\begin{array}{cc}v_{o d i 0} & v_{o q i 0} \\ v_{o q i 0} & -v_{o d i}\end{array}\right]$

$B_{V S I i}=\left[\begin{array}{cccc}-R_{v i} & -\omega_{0} L_{v i} & F_{i}-k_{P v i} R_{v i} & -k_{P v i} \omega_{0} L_{v i} \\ \omega_{0} L_{v i} & -R_{v i} & k_{P v i} \omega_{0} L_{v i} & F_{i}-k_{P v i} R_{v i}\end{array}\right]^{T}$

$B_{L C f i}=\left[\begin{array}{cccc}\frac{F_{i} k_{P c i}-k_{P c i} k_{P v i} R_{v i}}{L_{f i}} & \frac{-k_{P c i} k_{P v i} \omega_{0} L_{v i}}{L_{f i}} & \frac{-1}{C_{f i}} & 0 \\ \frac{k_{P c i} k_{P v i} \omega_{0} L_{v i}}{L_{f i}} & \frac{F_{i} k_{P c i}-k_{P c i} k_{P v i} R_{v i}}{L_{f i}} & 0 & \frac{-1}{C_{f i}}\end{array}\right]^{T}$

$A_{D G \mathrm{i}}$ and $A_{D G i j}$ are given in Appendix. In order to derive $A_{D G i}$ and $A_{D G i j}$, the current and power through the power network are linearized around the equilibrium point. To this end, partial derivatives of power network equations are obtained in terms of phase angle and voltage magnitude at the end nodes of interconnecting power line, which are the state variables determined by droop control signals.

4) Supplementary loop model: the additional control loop for reactive power sharing adds one more state variable to each DG unit according to (27) as the following:

$$
\dot{\alpha}_{i}=v_{\text {odi }}-V_{B}
$$

where $v_{\text {odi }}$ is the direct component of $i^{\text {th }}$ DG unit's output voltage considered as the state variable in (49). $V_{B}$ is the voltage magnitude at PCC. In [37], $V_{B}$ is considered as an input to individual parallel DG models. However, since its dynamics is determined by the dynamics of all the droop controllers in the MG (it is not a slack bus in the islanded MGs), it could not be considered as an input in isolated MGs. So it should be modeled by system variables. For the MG main bus, the shunt capacitor (compensator) model is obtained as:

$$
\begin{aligned}
& \dot{v}_{d B}=\omega_{0} v_{q B}-\frac{1}{C_{B}} \sum_{j=1}^{n} \sigma_{B j} i_{d B j}-\frac{1}{C_{B}} i_{L D C o n d B} \\
& \dot{v}_{q B}=-\omega_{0} v_{d B}-\frac{1}{C_{B}} \sum_{j=1}^{n} \sigma_{B j} i_{q j B}-\frac{1}{C_{B}} i_{L D C o n q B}
\end{aligned}
$$

where the index $B$ denotes the MG main bus and $C_{B}$ is the capacitance of the shunt compensator. $v_{d B}$ is the direct 
component of MG bus voltage $\left(V_{B}\right)$ and its dynamic is determined by injecting current from adjacent buses $\left(i_{B j}\right)$ and is modeled by (54). As described before (54) models the network current as a function of the voltage magnitude and phase angle of network buses, which are determined by the droop controllers. In this way, droop controllers' effect on voltage dynamic at bus B (PCC) is realized. The phase angle dynamic at bus $\mathrm{B}$ is needed which is obtained as:

$$
\delta_{B}=\arctan \left(v_{q B} / v_{d B}\right)
$$

Eqs. (69)-(70) add $n+2$ more state variables to the MG statespace model in (57). Consequently, $v_{\text {odref }}(41)$ at generation bus $i$ is updated by considering the modified droop control in (72) to obtain the augmented state-space model.

$$
v_{\text {odrefi }}=V_{0}-k_{q i} Q_{i}+k_{s i} \alpha_{i}-R_{v i} i_{o d i}+\omega_{0} L_{v i} i_{o q i}
$$

\begin{tabular}{|c|c|c|c|}
\hline LC filter & $\begin{array}{c}\boldsymbol{R}_{f} \\
0.1 \Omega \\
\end{array}$ & $\begin{array}{c}\boldsymbol{L}_{f} \\
1 \mathrm{e}-3 \mathrm{H} \\
\end{array}$ & $\begin{array}{c}C_{f} \\
50 \mathrm{e}-6 \mathrm{~F}\end{array}$ \\
\hline \multirow{2}{*}{$\begin{array}{c}\text { Voltage } \\
\text { control loop }\end{array}$} & $\overline{k_{P v}}$ & $\overline{k_{I V}}$ & $\bar{F}$ \\
\hline & 0.5 & 390 & 0.75 \\
\hline \multirow{2}{*}{$\begin{array}{c}\text { Current } \\
\text { control loop }\end{array}$} & $k_{P_{c}}$ & $k_{I c}$ & - \\
\hline & 10.5 & 16000 & - \\
\hline \multirow[t]{2}{*}{$\begin{array}{c}\text { Power } \\
\text { converter }\end{array}$} & $\begin{array}{l}\text { Switching } \\
\text { frequency }\end{array}$ & Rated power & Voltage (L-L) \\
\hline & $8 \mathrm{kHz}$ & $30 \mathrm{~kW}$ & $400 \mathrm{~V}$ \\
\hline
\end{tabular}

Table 1. Electrical and control parameters of VSI

\section{Simulation Results}

A meshed MG including three DG units is simulated in MATLAB/Simulink, Simscape toolbox. The simulated MG is shown in Fig. 4. The VSI control system used in the simulations is depicted in Fig. 5. The electrical and control parameters, related to LC filter and inner control loops, are given in Table 1. First, eigenvalue loci of the MG is drawn from the developed state-space model for eigenvalue analysis in order to evaluate the system stability. Then performance of the proposed supplementary loop in overcoming the drawbacks of conventional droop controls in reactive power sharing and voltage regulation is evaluated through different case studies. The performance of the proposed method is also compared with a consensus control [32]-[36].

Eigenvalue-analysis: dominant eigenvalue loci of the MG is depicted in Fig. 6. In order to emphasize the importance of the developed small-signal model, dominant eigenvalue loci of a meshed MG is compared with those of an MG with a parallel topology (all VSIs are connected to the PCC) both with the same electrical and control parameters. It is a wellknown fact that the dominant eigenvalues of the droop-based MG are related to droop loops [37]. Since the interaction of droop controllers is high in networked MGs through the power network, increasing droop gains pushes the control system to the unstable region, see Fig. 6(a), due to its narrow stability margin. While in MGs with parallel topology, dominant eigenvalues related to $V$ - $Q$ droop loop are located far in the left half s-plane, as shown in Fig. 6(b), and thus stability is a less important issue than in networked MGs. So it is critical to take stability into account in adjusting control parameters in networked MGs.

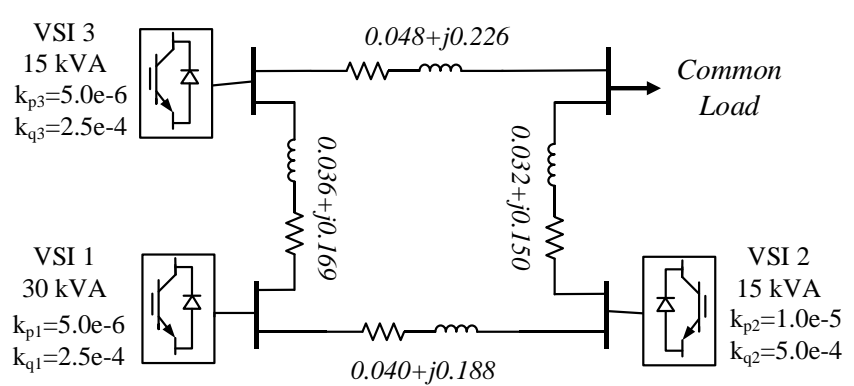

Fig. 4. Simulated MG topology

To this end, the developed small-signal model, by which the interaction of droop controllers has been properly modelled, is utilized to select appropriate supplementary gain. The supplementary loop adds a stable pole on the real axis closed to the imaginary axis as shown in Fig. 6(c). Increasing the corresponding control gain moves the pole toward the left side, which increases the response speed. On the other hand, it moves the conjugate poles related to the $V$ - $Q$ droop control loop to the right side, which makes the system oscillatory. Hence, a compromising should be established between response speed and oscillation.

Case study 1: in this case, the performance of the developed control method is evaluated in meshed MGs which is given in Fig. 4. Figs. 7(a) and 7(b) indicate disappointing performance of the conventional droop control in reactive power sharing and voltage regulation, whereas the suggested method implements reactive power sharing and voltage regulation properly, as shown in Figs. 8(a) and 8(b). Since voltage is a local variable, the voltage profile in the MG is restored (regulated) to the permitted band, rather than restoring the voltage magnitude at all the buses to a nominal value, which would be in conflict with reactive power sharing. Fig. 8(c) shows S\&H blocks that are triggered at the same time, which ensures accurate $Q$ sharing.

Comparison with consensus control: the effectiveness of the proposed method is compared with consensus-based method with the following consensus protocol [32]:

$$
\beta_{i}=k_{C i} \sum_{j=1}^{n} \sigma_{i j} \int\left(n_{i} Q_{i}-n_{j} Q_{j}\right)
$$

where $\beta$ is the consensus signal and $k_{C}$ is the consensus gain. Simulation results related to the consensus control method is given in Figs. 9(a) and (b).

As per the simulation results, advantages of the proposed method over the consensus method are: 1) Response speed of the proposed method is faster than the consensus one (three times faster according to Figs. 8(a) \& 9(a). 2) The consensus method needs higher bandwidth than the proposed one, since in the proposed method only a $\boldsymbol{D C}$ value (voltage) is send through the LBWCL to DG units, while in the consensus control, DG units exchange (send \& receive) reactive power information with their neighbours. Besides, frequency of reactive power oscillation is larger than voltage. 3) In the proposed method, voltage deviation is restored automatically, while in the consensus method, extra protocol is needed for voltage regulation, which in turn, requires higher bandwidth communication links. 4) The proposed method is more reliable because of the proposed voltage estimation method. 


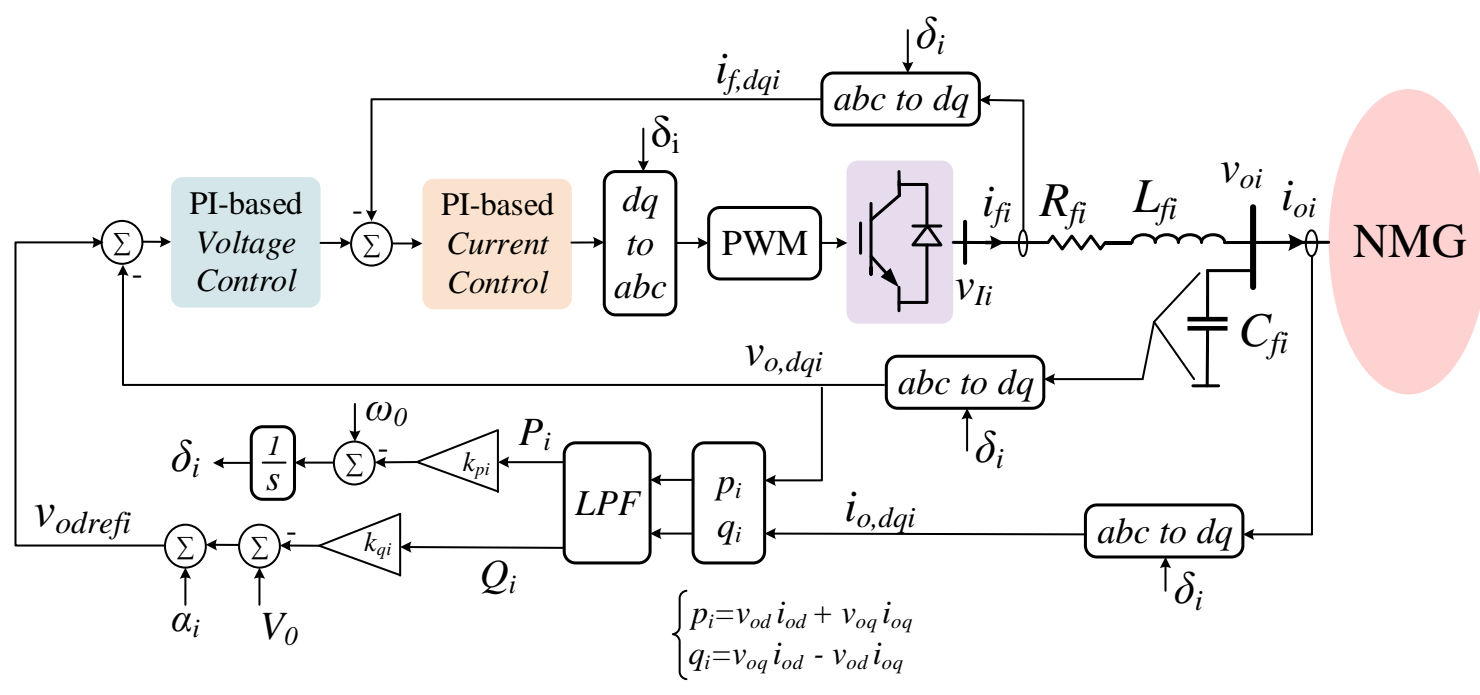

$a$

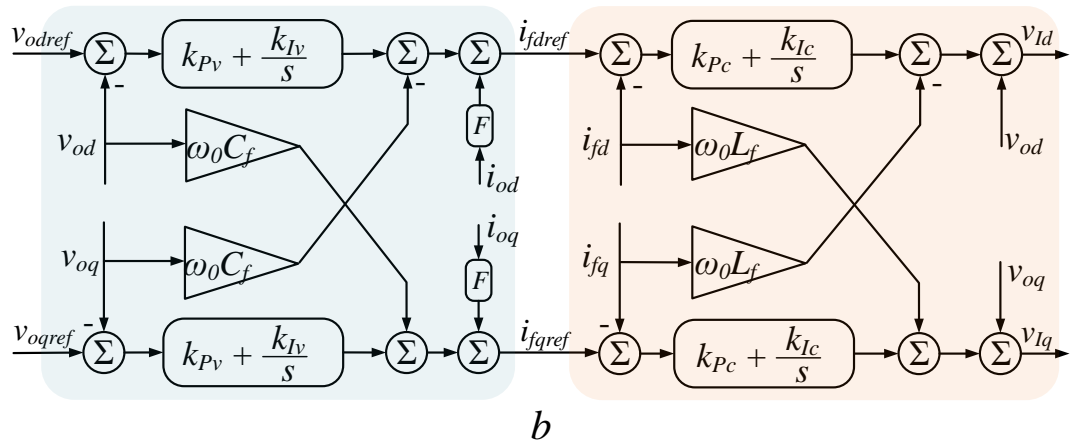

Fig. 5. VSI's control system adopted in the simulation, (a) the overall control system including power (droop) control loops and, (b) inner control loops

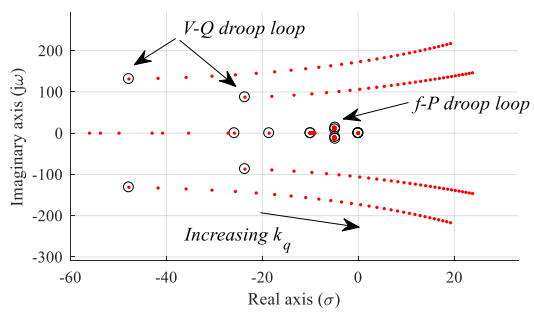

$a$

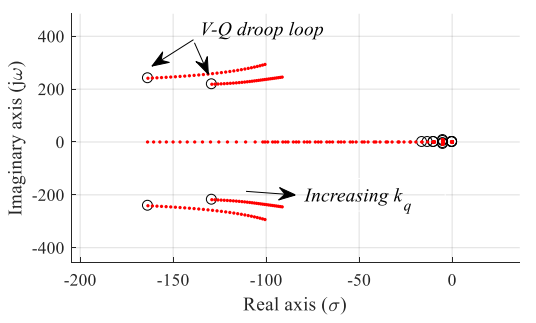

$b$

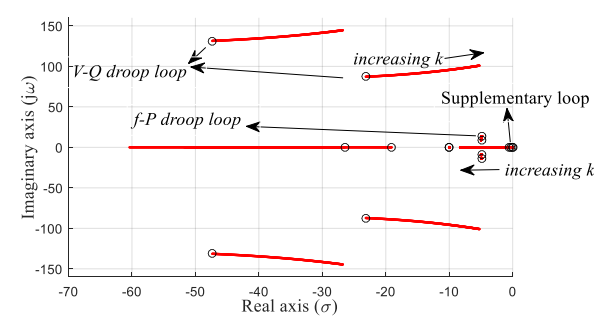

$c$

Fig. 6. Eigenvalue loci, (a) dominant eigenvalue loci of the $M G$ with meshed (networked) topology where increasing $V$ - $Q$ droop gains pushes the control system to the unstable region due to the interaction of droop controllers, (b) dominant eigenvalue loci of a parallel-based $M G,(c)$ dominant eigenvalue loci of the $M G$ with the proposed supplementary loop
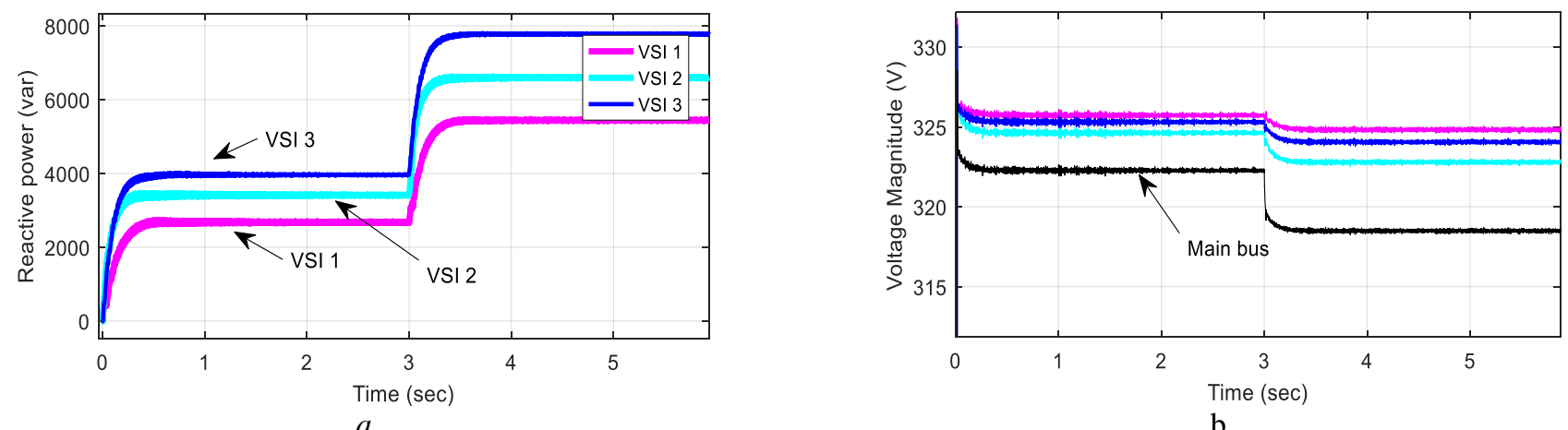

Fig. 7. Simulation results for the conventional droop control: (a) reactive power sharing, (b) voltage drop 

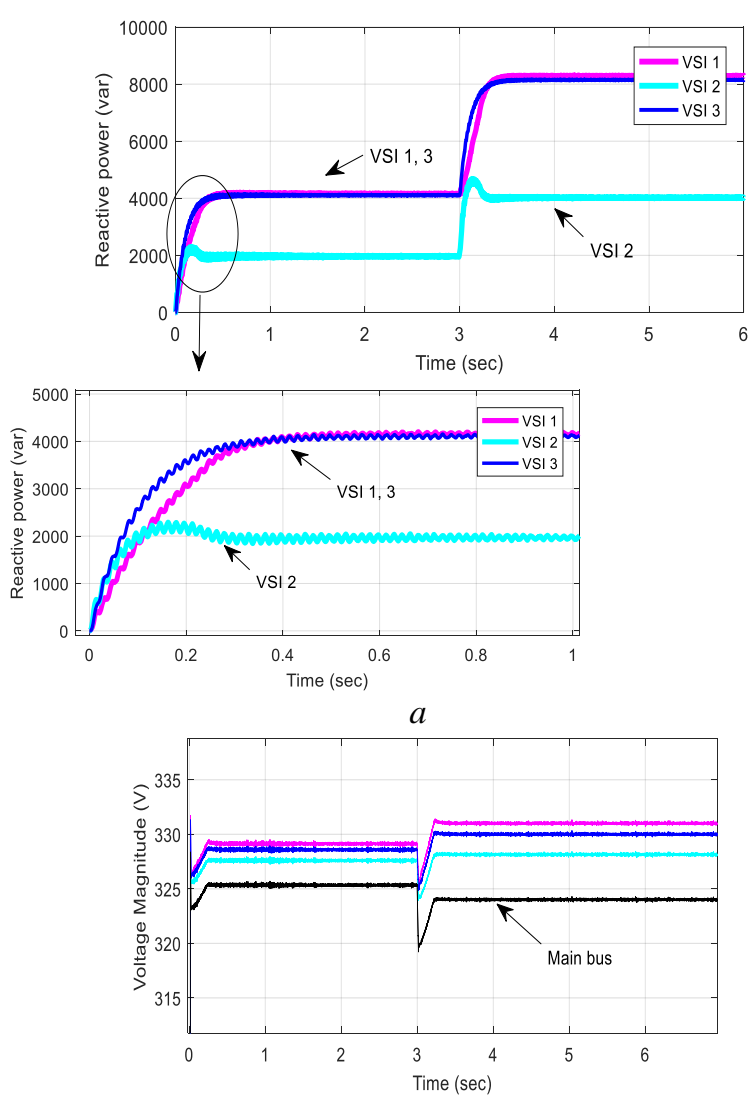

$b$

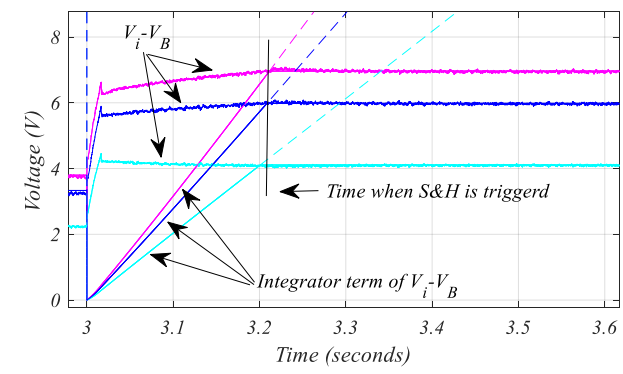

$c$

Fig. 8. Simulation results for the proposed control method: (a) reactive power sharing, (b) voltage drop, (c) the $S \& H$ unit performance

Case study 2: in this case the performance and robustness of the proposed method is evaluated whenever the MG is exposed to a large disturbance. In order to insert a large disturbance to MG, DG 2 is turned off and on at $t=2(s)$ and $t=4(s)$, respectively, as shown in Fig. 10. When DG 2 is disconnected from the MG, DG 1 \& DG 3 are responsible for supporting the reactive power demanded by loads. Since both DGs (1\&3) have equal droop gains, their contribution in supporting reactive power is equal which is properly implemented by the control method. After reconnection of DG 2, all DG units support the reactive power proportional to their assigned droop gains while the stability of the autonomous MG is secured thanks to the proposed method which is robust in a wide range of operating points. The voltage regulation is also successfully implemented, as shown in Fig. 10(b). When DG 2 is disconnected, its output voltage moves to the nominal value $\left(V_{0}=326.6 \mathrm{~V}\right)$ due to its zero output reactive power. After reconnection, the voltage magnitude is regulated in the nominal band, in accordance to voltage at other buses, so that accurate reactive power sharing is achieved.
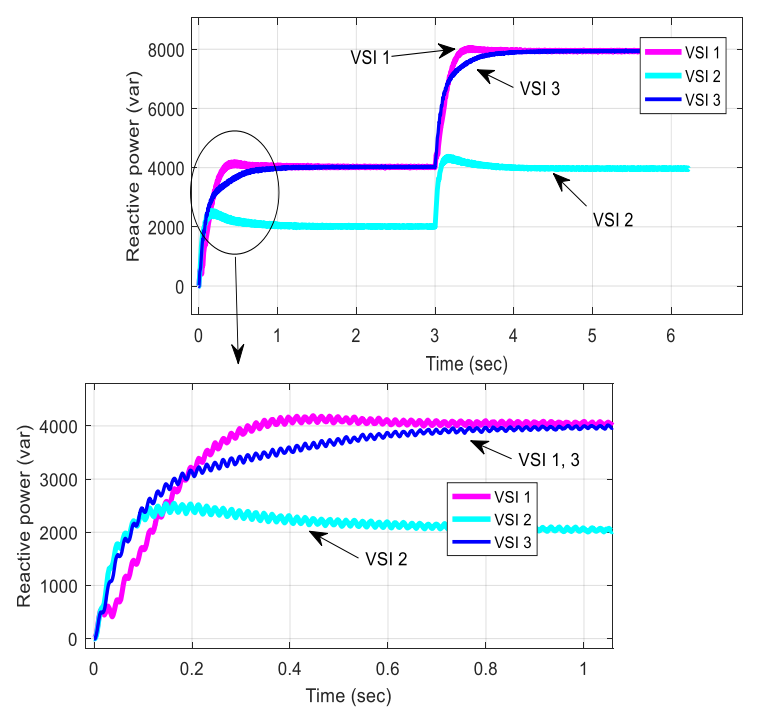

$a$

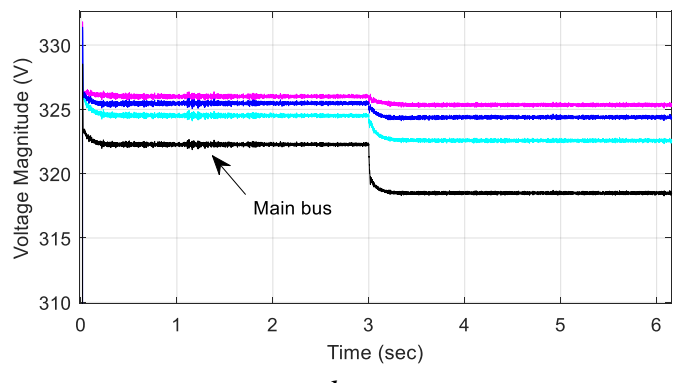

b

Fig. 9. Simulation results for the consensus control, (a) reactive power sharing, $(b)$ voltage drop

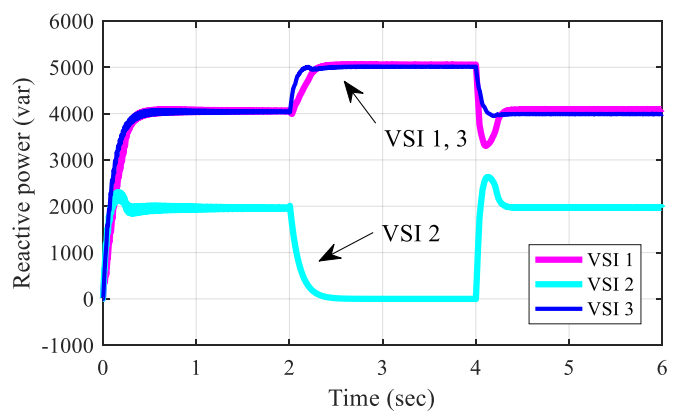

a

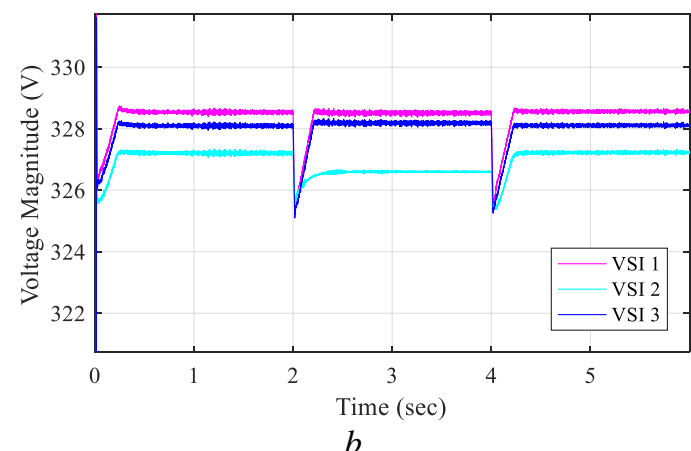

Fig. 10. Simulation results for the proposed control method in response to large disturbances (DG 2 is turned offlon at $t$ $=2(s)$ and $t=4(s)$, respectively): (a) reactive power sharing, (b) voltage regulation 


\section{Conclusion}

This paper deals with the poor performance of the $V$ - $Q$ droop control loop in reactive power sharing and voltage regulation in autonomous networked MGs. A power flowbased approach has been developed to estimate the voltage at generation buses as well as PCC in the isolated droopcontrolled MGs from DG units' point of view. The powerflow method revealed that the droop control loop operation leads to poor power quality at generation buses as well as a drastic voltage drop at PCC. Then, a novel control strategy, as a supplementary loop to the droop loop, has been proposed to overcome the $V-Q$ droop controller drawbacks. By adopting the proposed method, reactive power sharing is established fast and precisely, while, power quality is improved throughout the MG by restoring the voltage to its nominal band simultaneously. Simulation results verified the effectiveness and superiority of the proposed method over the consensus control.

Dynamic stability is also assessed by developing a novel state-space model of MG including the power network and interaction of droop controllers through it. Nevertheless, developing a reduced-order model to be adopted in the synthesis of advanced control systems for improving robustness of the method remains as an open topic which is considered as the authors' future work.

\section{Acknowledgments}

Part of this work is supported by National Natural Science Foundation of China (51777078).

\section{References}

[1] Moradi, M. H., \& Eskandari, M.: 'A hybrid method for simultaneous optimization of DG capacity and operational strategy in microgrids considering uncertainty in electricity price forecasting', Renewable energy, 68, 697-714

[2] Yang, B., Yu, T., Shu, H., et al.: 'Robust sliding-mode control of wind energy conversion systems for optimal power extraction via nonlinear perturbation observers', App. Energy, 2018, 210, 711-723

[3] Yang, B., Yu, T., Shu, H., et al.: 'Passivity-based linear feedback control of permanent magnetic synchronous generator-based wind energy conversion system: design and analysis', IET Renew. Power Gen., 2018, 12(9), 981-991

[4] Yang, B., Yu, T., Shu, H., et al.: 'Perturbation observer based fractional-order PID control of photovoltaics inverters for solar energy harvesting via Yin-Yang-Pair optimization', Energy Conv. and Manag., 2018, 171, 170-187

[5] Yang, B., Yu, T., Shu, H., et al.: 'Energy reshaping based passive fractional-order PID control design and implementation of a gridconnected PV inverter for MPPT using grouped grey wolf optimizer', Solar Energy, 2018, 170, 31-46

[6] Olivares, D.E., Mehrizi-Sani, A., Etemadi, A.H., et al.: 'Trends in microgrid control', IEEE Trans. Smart Grid, 2014, 5(4), pp. 19051919

[7] Hatziargyriou, N., Asano, H., Iravani, R., Marnay. C.: 'Microgrids', IEEE Power and Energy Magazine, 2007, 5(4), pp. 78-94

[8] Moradi, M.H., Eskandari, M., Hosseinian, S.M.: 'Operational strategy optimization in an optimal sized smart microgrid', IEEE Trans. Smart Grid, 2015, 6(3), pp. 1087-1095

[9] Moradi, M.H., Eskandari, M., Hosseinian, S.M.: 'Cooperative control strategy of energy storage systems and micro sources for stabilizing microgrids in different operation modes, " Int. J. Electr. Power Energy Syst., 2016, 6, pp. 390-400
[10] Moradi, M.H., Eskandari, M., Siano, P.: 'Safe transition from connection mode to islanding mode in Microgrids', IEEE, Electrical Engineering (ICEE), 2016 24th Iranian Conference on, pp. 1902-1907

[11] Moreira, C.L., Resende, F.O., Lopes, J.P.: 'Using low voltage microgrids for service restoration', IEEE Trans. On Power Syst. 2007, 22(1), pp. 395-403.

[12] Rocabert, J., Luna, A., Blaabjerg, F., Rodríguez, P.: 'Control of power converters in AC microgrids,' IEEE Trans. On Power Electron., 2012, 27(11), pp. 4734-4749

[13] Guerrero, J.M., Vasquez, J.C., Matas, J.: 'Hierarchical control of droop controlled AC and DC microgrids-A general approach toward standardization', IEEE Trans. on Ind. Electron., 2011, 58(1), pp. 158172

[14] Eskandari, M., Li, L., Moradi, M. H., Siano, P., Blaabjerg, F.: 'Active Power Sharing and Frequency Restoration in an Autonomous Networked Microgrid' IEEE Trans. on Power Syst., 2019

[15] Zandi, F., Fani, B., Sadeghkhani, I., Orakzadeh, A.: 'Adaptive complex virtual impedance control scheme for accurate reactive power sharing of inverter interfaced autonomous microgrids,' IET Gen. Trans. \& Dist., 2018, 12(22), pp. 6021-6032

[16] Chen, Y., Guerrero, J. M., Shuai, Z., Chen, Z., Zhou, L., \& Luo, A.: 'Fast reactive power sharing, circulating current and resonance suppression for parallel inverters using resistive-capacitive output impedance', IEEE Trans. on Power Electron., 2015, 31(8), pp. 55245537.

[17] H. Han, Y. Liu, Y. Sun, M. Su, J. M. Guerrero, “An Improved Droop Control Strategy for Reactive Power Sharing in Islanded Microgrid', IEEE Trans. on Power Electron., 2015, 30(6), pp. 3133-3141

[18] Kosari, M., Hosseinian, S.H.: 'Decentralized reactive power sharing and frequency restoration in islanded microgrid', IEEE Trans. on Power Syst., 2017, 32(4), pp. 2901-2912

[19] Kim, J. W., Choi, H. S., Cho, B. H.: 'A novel droop method for converter parallel operation', IEEE Trans. on Power Electron., 2002, 17(1), pp. 25-32

[20] Zhu, Y., Zhuo, F., Wang, F., Liu, B., Gou, R., Zhao, Y.: 'A virtual impedance optimization method for reactive power sharing in networked microgrid', IEEE Trans. on Power Electron., 2015, 31(4), pp. 2890-2904

[21] Wang, Y., Wang, X., Chen, Z., Blaabjerg, F.: 'Distributed optimal control of reactive power and voltage in islanded microgrids', IEEE Trans. on Ind. Appl., 2017, 53(1), pp. 340-349

[22] Wang, Y., Chen, Z., Wang, X., et al.: 'An Estimator-Based Distributed Voltage-Predictive Control Strategy for AC Islanded Microgrids', IEEE Trans. on Power Electron., 2015, 30(7), pp. 3934-3951

[23] Zhang, J., Shu, J., Ning, J., et al.: 'Enhanced proportional power sharing strategy based on adaptive virtual impedance in low-voltage networked microgrid,' IET Gen. Trans. \& Dist., 2018, 12(11), pp. 2566- 2576

[24] Alizadeh, E., Birjandi, A.M., Hamzeh, M.: 'Decentralised power sharing control strategy in LV microgrids under unbalanced load conditions,' IET Gen. Trans. \& Dist., 2017, 11(7), pp. 1613-1623

[25] Eskandari, M., Li, L.: 'Microgrid operation improvement by adaptive virtual impedance', IET Renew. Power Gen., 2018, 13(2), 296-307

[26] Sao, C.K., Peter W. Lehn, P.W.: 'Autonomous load sharing of voltage source converters' IEEE Trans. on Power Del., 2005, 20(2), pp. 10091016

[27] Yun Wei Li, Ching-Nan Kao, 'An accurate power control strategy for power-electronics-interfaced distributed generation units operating in a low-voltage multi-bus microgrid', IEEE Trans. on Power Electron., 2009, 24(12), pp. 2977-2988

[28] Rokrok, E., Golshan, M.E.H.: 'Adaptive voltage droop scheme for voltage source converters in an islanded multibus microgrid', IET Renew. Power Gen., 2010, 4(5), pp. 562-578

[29] Yao, W., Chen, M., Matas, J., Guerrero, J. M., \& Qian, Z. M.: 'Design and analysis of the droop control method for parallel inverters considering the impact of the complex impedance on the power sharing', IEEE Trans. on Ind. Electron., 2011, 58(2), pp. 576-588

[30] Lee, C. T., Chu, C. C., Cheng, P. T.: 'A new droop control method for the autonomous operation of distributed energy resource interface converters', IEEE Trans. on Power Electron., 2012, 28(4), 1980-1993

[31] Eskandari, M. Li, L., Moradi, M.H.: 'Decentralized optimal servo control system for implementing instantaneous reactive power sharing in microgrids', IEEE Trans. on Sustain. Energy, 2018, 9(2), pp. 525537

[32] Shafiee, Q., Guerrero, J.M., \& Vasquez, J.C.: 'Distributed secondary control for islanded microgrids-A novel approach', IEEE Trans. on Power Electron., 2013, 29(2), 1018-1031 
[33] Simpson-Porco, J.W., Shafiee, Q., Dörfler, F., Vasquez, J.C., Guerrero, J.M., \& Bullo, F.: 'Secondary frequency and voltage control of islanded microgrids via distributed averaging, IEEE Trans. on Ind. Electron., 62(11), 7025-7038

[34] Lu, L.Y., Chu, C.C.: 'Consensus-based droop control synthesis for multiple DICs in isolated micro-grids', IEEE Trans. on Power Syst., 2014, 30(5), 2243-2256

[35] Zhang, H., Kim, S., Sun, Q., Zhou, J.: 'Distributed Adaptive Virtual Impedance Control for Accurate Reactive Power Sharing Based on
Consensus Control in Microgrids', IEEE Trans. Smart Grid, 2016, 6(3), pp. 1087-1095

[36] Schiffer, J., Seel, T., Raisch, J., Sezi, T.: 'Voltage stability and reactive power sharing in inverter-based microgrids with consensus-based distributed voltage control. IEEE Trans. on Cont. Syst. Tech., 2015, 24(1), pp. 96-109

[37] Pogaku, N., Prodanovic', M., Green, T.C.: 'Modeling, Analysis and Testing of Autonomous Operation of an Inverter-Based Microgrid', IEEE Trans. Power Electron., 2007, 22(2), pp. 613-625

\section{Appendix}

\section{Jacobian Matrices}

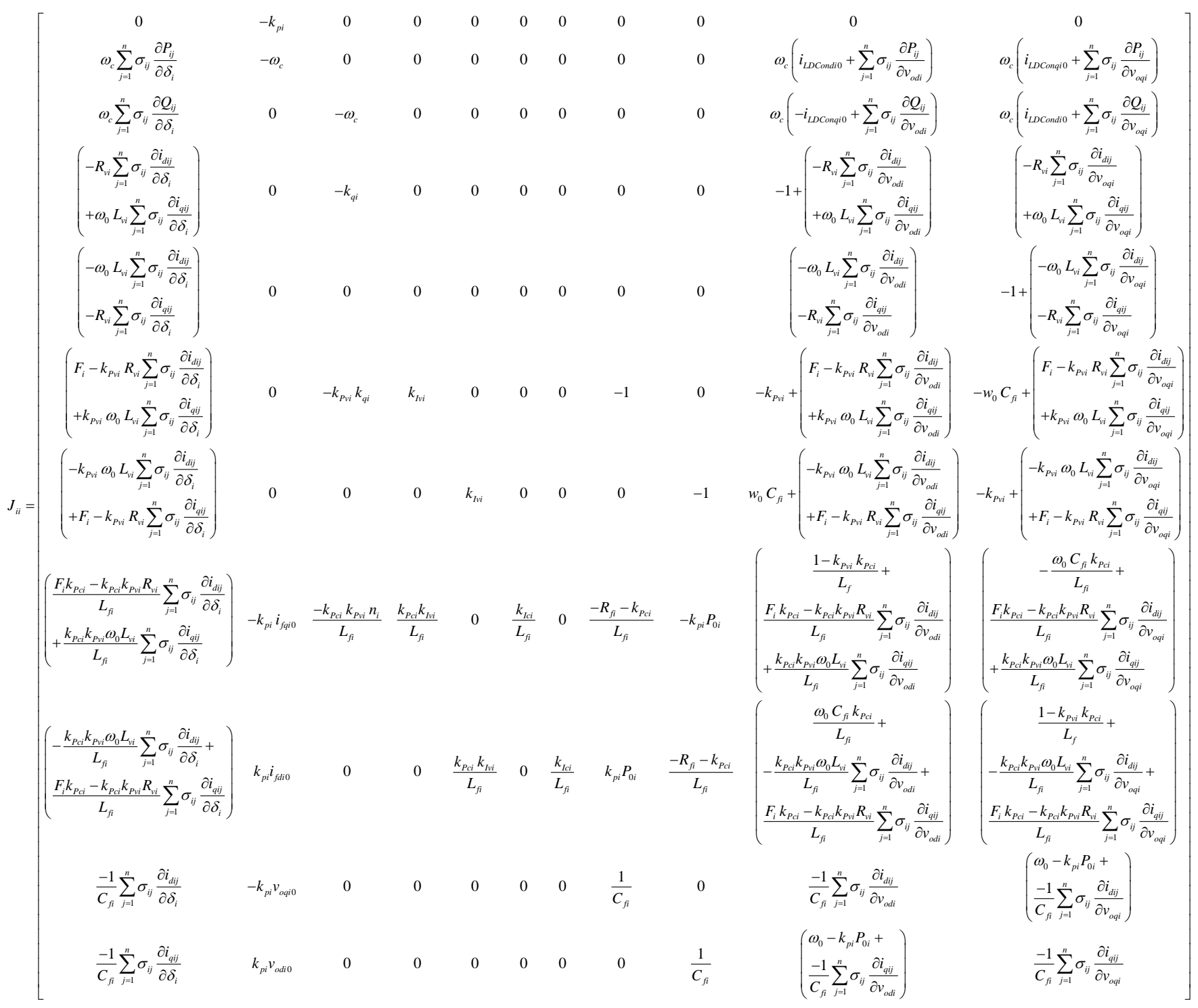




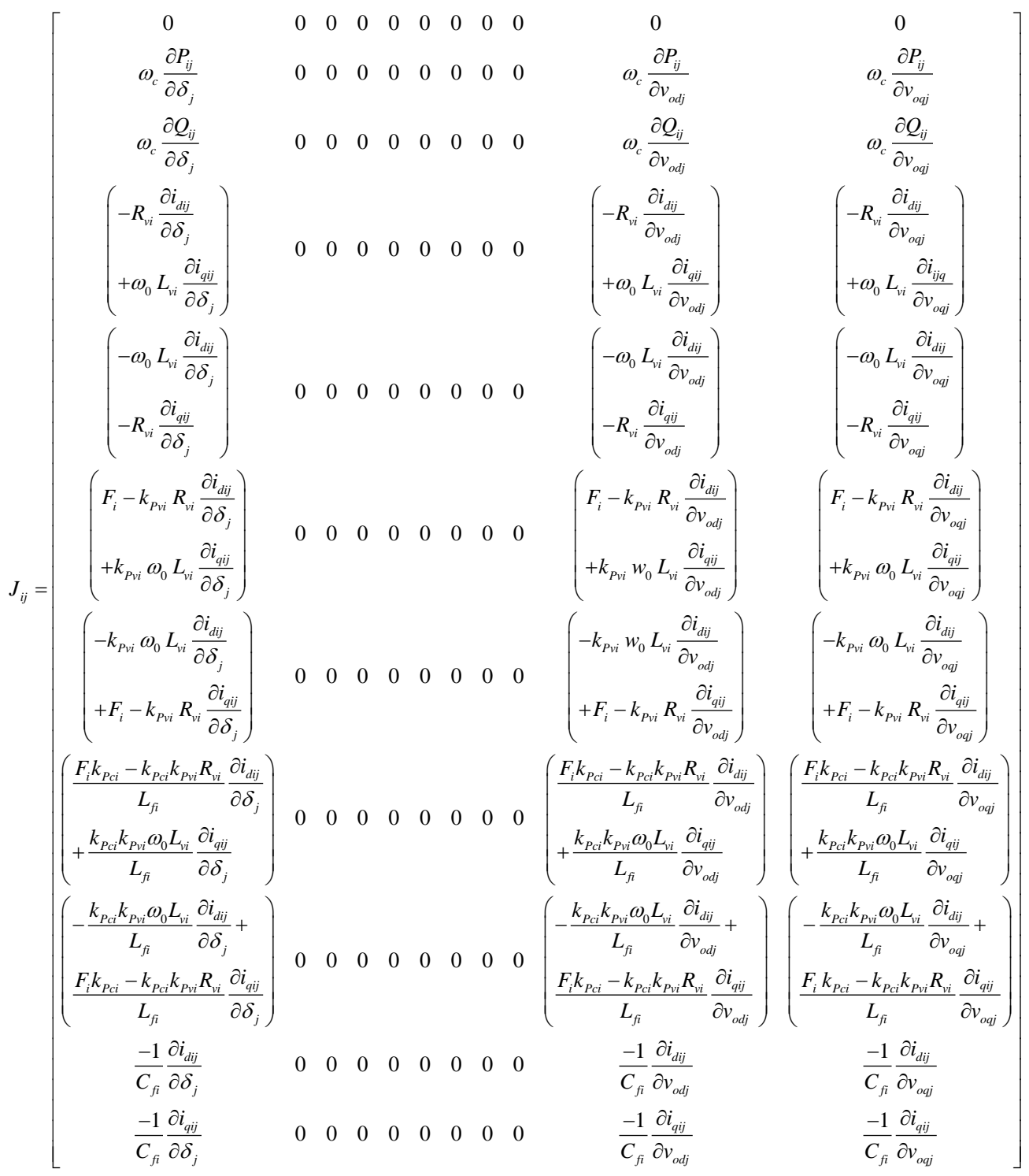

